OPEN ACCESS

Edited by:

Yuzhen $X u$,

Tongji University, China

Reviewed by:

Yongjun Cao,

The Second Affiliated Hospital of

Soochow University, China

Taizen Nakase,

Tohoku University, Japan

*Correspondence:

Yumin $\mathrm{Li}$

liym@|zu.edu.cn

tThese authors have contributed equally to this work and share first

authorship

Specialty section:

This article was submitted to

Neurological Biomarkers,

a section of the journal

Frontiers in Neurology

Received: 15 September 2021

Accepted: 07 October 2021

Published: 25 November 2021

Citation:

Liu Y, Li X, Song F, Yan X, Han Z,

Tang $F$ and Li Y (2021) Clinical

Features and Prognostic Factors of Acute Ischemic Stroke Related to Malignant Gastrointestinal Tumor

Front. Neurol. 12:777483

doi: 10.3389/fneur.2021.777483

\section{Clinical Features and Prognostic Factors of Acute Ischemic Stroke Related to Malignant Gastrointestinal Tumor}

\author{
Yating Liu ${ }^{1,2 \dagger}$, Xin Li ${ }^{3 \dagger}$, Feixue Song ${ }^{1}$, Xin Yan ${ }^{2}$, Zhijian Han ${ }^{2}$, Futian $\mathrm{Tang}^{2}$ and Yumin $\mathrm{Li}^{2 *}$ \\ ${ }^{1}$ Department of Oncology, Lanzhou University Second Hospital, Lanzhou, China, ${ }^{2}$ Key Laboratory of the Digestive System \\ Tumors of Gansu Province, Lanzhou University Second Hospital, Lanzhou, China, ${ }^{3}$ Department of Neurology, Lanzhou \\ University Second Hospital, Lanzhou, China
}

Objectives: To analyze the clinical and imaging features of acute ischemic stroke (AIS) related to gastrointestinal malignant tumor, and to explore the prognostic factors.

Methods: Clinical data of consecutive patients with gastrointestinal malignant tumor complicated with AIS admitted to the Department of Neurology and Oncology in Lanzhou University Second Hospital from April 2015 to April 2019 were retrospectively analyzed. Patients were divided into good prognosis (mRS 0-2) and poor prognosis ( $m R S>2$ ) based on a 90-day mRS score after discharge. The multivariate logistic regression model was used to analyze the prognostic factors.

Results: A total of 68 patients were enrolled with an average age of $61.78 \pm 6.65$ years, including 49 men (72.06\%). There were 18 patients in the good prognosis group and 50 patients in the poor prognosis group. The univariate analysis showed that Hcy, D-dimer, thrombin-antithrombin complex (TAT), and three territory sign in magnetic resonance imaging (MRI) were the risk factors for poor prognosis. Multivariate analysis showed that increased D-dimer (OR 4.497, 95\% Cl 1.014-19.938) and TAT levels (OR 4.294, 95\% Cl 1.654-11.149) were independent risk factors for the prognosis in such patients.

Conclusion: Image of patients with gastrointestinal malignant tumor-related AIS is characterized by three territory sign (multiple lesions in different vascular supply areas). Increased TAT and D-dimer levels are independent prognostic risk factors. TAT is more sensitive to predict prognosis than D-dimer.

Keywords: gastrointestinal malignant tumor, acute ischemic stroke, D-dimer (DD), TAT, prognosis

\section{INTRODUCTION}

Armand Trousseau first reported the relation between thrombosis and malignant tumors in 1865 (1). Subsequent studies have confirmed that thrombosis is a common complication of malignant tumors, which is the second leading cause of death in patients with cancer (2). Thrombotic complications of cancer include arterial or venous thromboembolism and disseminated intravascular coagulation (DIC) $(3,4)$. Up to $15 \%$ of patients with malignant tumors have a history of acute ischemic stroke (AIS) (5), and about $20 \%$ of patients with AIS 
due to an unknown cause (cryptogenic stroke) may have latent malignant tumors (6). Previous studies on gastrointestinal malignant tumor-related thromboembolism mainly focused on venous thromboembolism (7), and clinical studies on the associated AIS focused on a single type of gastrointestinal tumor (8), leading to a paucity of systematic analysis of different gastrointestinal tumor types. The present work retrospectively analyzes the 5-year clinical data of consecutive patients with gastrointestinal malignant tumor-related AIS in a single center. Prognostic factors are analyzed to improve clinical understanding of the gastrointestinal malignant tumorrelated AIS.

\section{THE MECHANISMS OF MALIGNANT TUMOR-RELATED AIS}

Abnormal coagulation function is the main cause of AIS in patients with malignant tumor. Abnormal cerebrovascular coagulation is seen in patients with malignant tumors including breast cancer, leukemia, and lymphoma, after performing autopsy, which indicated the association between malignant tumors and coagulation and thrombosis (9). Kim et al. (10) conducted a prospective study and found that Ddimer levels in tumor-related cerebral infarction patients were significantly higher with increased incidence of multifocal cerebral infarction compared with conventional stroke risk factors. Tumor onset and progression are often accompanied by hypercoagulability, resulting in systemic and cerebral-arterial or venous thrombosis (11).

Adenocarcinomas, especially those of the pancreas, colon, breast, lung, prostate, and ovary induce thrombosis by producing and releasing mucin (a high-molecular weight molecule that is glycosylated and secreted by the endothelial cells) directly into the blood, which promotes a hypercoagulation state. Mucin can also interact with certain cellular adhesion molecules on endothelial cells, platelets, and lymphocytes, triggering the formation of microthrombi, which is rich in platelets (12). Meanwhile, tumor cells can produce cancer coagulants (cysteine protease that can independently activate coagulation $\mathrm{X}$ factor), tissue factors (binding with coagulation factor VII), and release inflammatory and vascular endothelial growth factors that can mediate coagulation and thrombosis (13-15). For example, tumor necrosis factor- $\alpha$ (TNF- $\alpha$ ) affects the anticoagulant properties of the vascular endothelial cells through tissue factors, which promotes thrombin production, fibrin clot formation, and fibrin deposition in blood vessels, and can reduce fibrinolysis by inhibiting tPA activity (16). Tumors may also be associated with acute or chronic DIC. Due to excessive activation of the coagulation process, the imbalance between coagulation and fibrinolysis will eventually lead to thrombosis and arterial occlusion, resulting in AIS (17). The binding of malignant tumor cells with certain nonspecific immune signaling molecules, such as selectin, chemokines, and the corresponding receptors, is conducive to tumor invasion, migration, and adhesion. This will damage cellular connection and cause endothelial injury (18). The activation of the host immune system and the release of inflammatory factors will damage vascular endothelium and thus promote thrombosis (19). Figure 1 shows the formation mechanism of hypercoagulable state in malignant tumors.

Nonbacterial thrombotic endocarditis (NBTE) caused by platelet thrombotic inflammatory complex is another important cause of malignant tumor-related AIS. In a work which consisted of 2,627 cases of postmortem examination, found that in 16 patients with malignant tumor complicated with NBTE, seven were complicated with cerebral infarction (20). This indicated that detaching of cardiac embolus in patients with malignant tumor complicated with NBTE can directly lead to AIS. In addition, infection $(21,22)$, tumor-related chemotherapy (23), endocrine therapy $(24,25)$ and, radiotherapy (26) can also lead to AIS in patients with a malignant tumor.

\section{MATERIALS AND METHODS Study Population}

The clinical baseline data of patients with gastrointestinal malignant tumor complicated with AIS admitted to the Department of Neurology and Oncology in Lanzhou University Second Hospital from April 2015 to April 2019, were retrospectively analyzed. Eligible patients were required to meet the following criteria: (1) patients aged $\geq 18$ years; (2) diagnosed with acute cerebral infarction conforming to Chinese Guidelines for the Diagnosis and Treatment of Acute Ischemic Stroke (2014) (5); (3) having clear imaging evidence in magnetic resonance imaging (MRI); (4) within 7 days of onset; (5) with a history of active malignant gastrointestinal tumor in the past or during follow-up. Patients were excluded if they had the following characteristics: (1) a history of TIA or cerebral hemorrhage; (2) primary tumors of the central nervous system, hematological, and other systems; (3) incomplete medical records; (4) poor prognosis due to tumor progression or chemotherapy.

\section{Study Measures \\ Baseline and MRI Data}

General information of the patients was retrieved, including gender, age, medical history and complications, laboratory tests on the second day of admission, tumor related data, and the results of echocardiography. The MRI data of the patients were collected. The location and the number of lesions on DWI were recorded. Lesions in both hemispheres were defined as bilateral infarction, and multiple infarctions referred to more than two lesions. Distribution patterns of the lesions were categorized into the following: (1) one territory sign: single or multiple lesions in unilateral anterior or posterior circulation; (2) two territory sign: single or multiple lesions in unilateral anterior and posterior circulations, or in bilateral anterior circulations; (3) three territory sign: bilateral single or multiple lesions in bilateral anterior and posterior circulations. Prognosis of the patients was evaluated by mRS, 90 days after discharge. An mRS score of $0-$ 2 was defined as good prognosis, and score $>2$ was regarded as poor prognosis. 


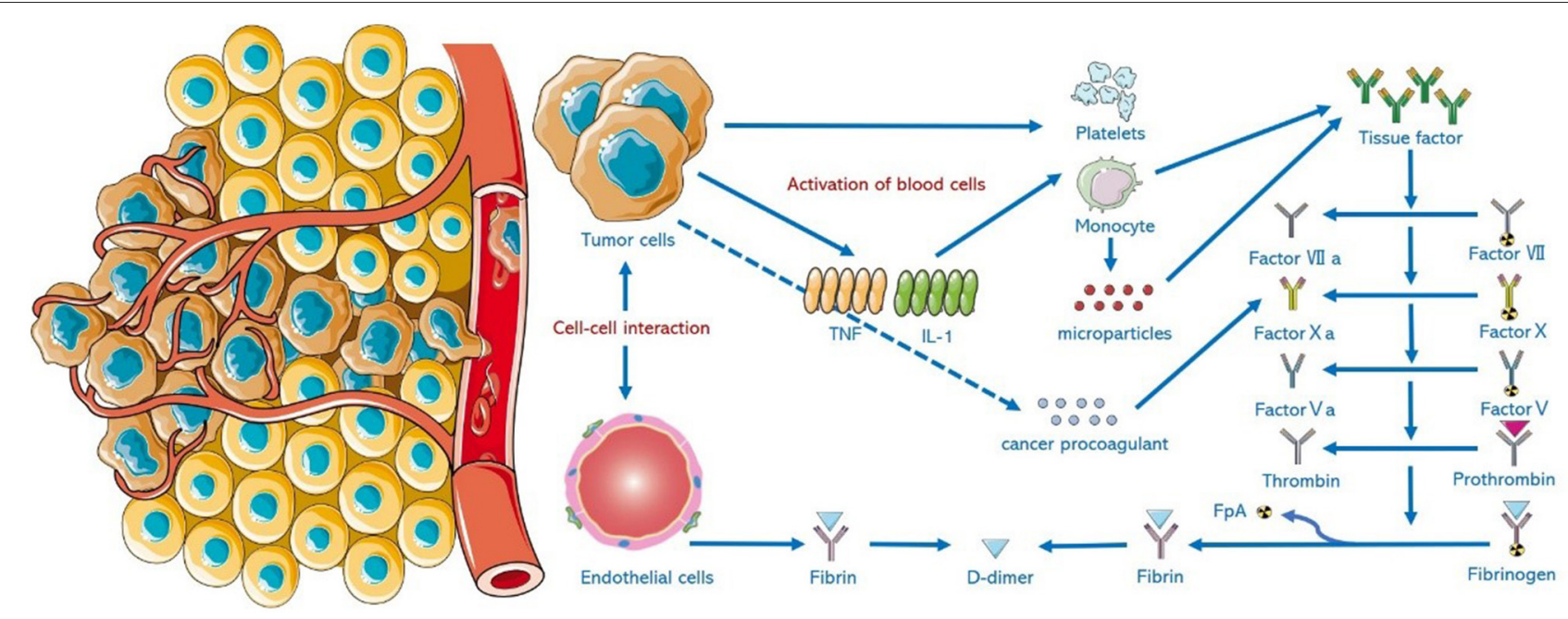

FIGURE 1 | Mechanism of hypercoagulable state in malignant tumors. Tumor cells activate cellular systems in vivo through intercellular interactions and injured endothelial cells. Tumor cells can directly release tissue factor (TF) and cancer procoaparticles (CP). Tumor cells produce cytokines including interleukin-1 (IL-1) and tumor necrosis factor (TNF). Activated blood cells (such as monocytes and platelets) and the microparticles (MP) produced by these cells work synergistically to increase TF expression and activate the coagulation system in vivo.

\section{Statistical Analysis}

Statistical analysis was performed by the SPSS 25.0. Enumeration data were recorded in ratio or proportion using $\chi 2$ or Fisher tests for comparisons among groups. Normally distributed measurement data was expressed as $\mathrm{X} \pm \mathrm{S}$, using $t$-test for comparisons among groups. Median and range (M, P25-P75) were used if the measurement data were not normally distributed, and the Mann-Whitney $U$ test was used for comparisons among groups. Variables with $P<0.05$ in univariate analysis were taken as independent variables with a 90-day prognosis as dependent variable. Multivariate logistic regression analysis was used to screen out independent risk factors for prognosis. $P<0.05$ was considered statistically significant.

\section{RESULTS}

A total of 68 patients with gastrointestinal cancer complicated with AIS were enrolled in this study, including 49 (72.09\%) men and 19 (27.91\%) women, with a mean age of $61.78 \pm$ 6.65 years. Cases of complications included hypertension, diabetes, hyperlipidemia, and coronary heart disease, and were 45 (66.18\%), 38 (55.88\%), 43 (63.24\%), and 45 (66.18\%), respectively. Patients with a history of drinking and smoking accounted for 50.00 and $35.29 \%$, respectively. Fifteen $(22.06 \%)$ cases and $14(20.59 \%)$ cases had a history of atrial fibrillation and stroke, respectively. The types of gastrointestinal malignant tumors included gastric cancer (42/68, 61.76\%), colorectal cancer $(17 / 68,25.00 \%)$, gallbladder cancer $(4 / 68,5.88 \%)$, pancreatic cancer $(2.68,2.94 \%)$, liver cancer $(2 / 68,2.94 \%)$, and esophageal cancer (1/68, 1.47\%). Adenocarcinoma was the main pathological tumor type, accounting for $63 / 68$ cases (92.65\%). Fifty-two (76.47\%) cases had a history of malignant tumor, 16 (23.53\%) cases without a previous history of tumor. Malignant tumors were diagnosed during hospitalization or follow-up due to AIS. MRI showed multiple scattered lesions. Patients with two and three territory signs accounted for 25 (36.76\%) and $30(44.12 \%)$ cases, respectively; one territory sign was only observed in seven $(10.29 \%)$ cases. Figure 2 shows the characteristics of gastrointestinal malignant tumor-related AIS in MRI.

The baseline characteristics of all study population and univariate regression analysis of prognosis are summarized in Table 1, with 18 patients in the good prognosis group and 50 patients in the poor prognosis group. Compared with the good prognosis group, patients in the poor prognosis group of three territory sign in MRI (52.00 vs. $22.22 \%, P=0.029)$ showed higher levels of $\mathrm{Hcy}(8.84 \pm 3.03$ vs. $17.00 \pm 3.40 \mu \mathrm{mol} / \mathrm{L}, P=0.047)$ and D-dimer $(2.12 \pm 0.78$ vs. $1.24 \pm 0.70 \mathrm{mg} / \mathrm{L}, P<0.001)$, and also higher levels of TAT $(8.07 \pm 3.99$ vs. $3.75 \pm 1.40 \mathrm{ng} / \mathrm{ml}, P<$ $0.001)$, all with significant differences $(P<0.05)$.

Table 2 shows the multivariate analysis of prognosis. The results showed higher levels of D-dimer (OR 4.497, 95\% CI 1.014-19.938) and TAT (OR 4.294, 95\% CI 1.654-11.149) which are independent risk factors for the prognosis in patients with gastrointestinal malignant tumor-related AIS $(P<0.05)$.

The baseline levels of D-dimer and thrombin-antithrombin (TAT) were $1.89 \pm 0.85$ and $6.93 \pm 3.98 \mathrm{ng} / \mathrm{ml}$, respectively. The levels of D-dimer at 3, 6, and 9-month follow-up were $1.65 \pm$ $0.80,1.52 \pm 0.97$, and $1.39 \pm 1.02 \mathrm{mg} / \mathrm{L}$, respectively; the levels of TAT were $8.46 \pm 6.17,9.88 \pm 8.61$, and $9.33 \pm 8.82 \mathrm{ng} / \mathrm{ml}$, respectively. An ROC curve analysis showed that when the AUC of D-dimer was 0.894 with a cutoff value of $1.60 \mathrm{mg} / \mathrm{L} \mathrm{FEU}$, the diagnostic sensitivity and specificity were 78.0 and $94.4 \%$, respectively; when the AUC of TAT was 0.926 with a cutoff value of $5.05 \mathrm{ng} / \mathrm{mL}$, the diagnostic sensitivity and specificity 


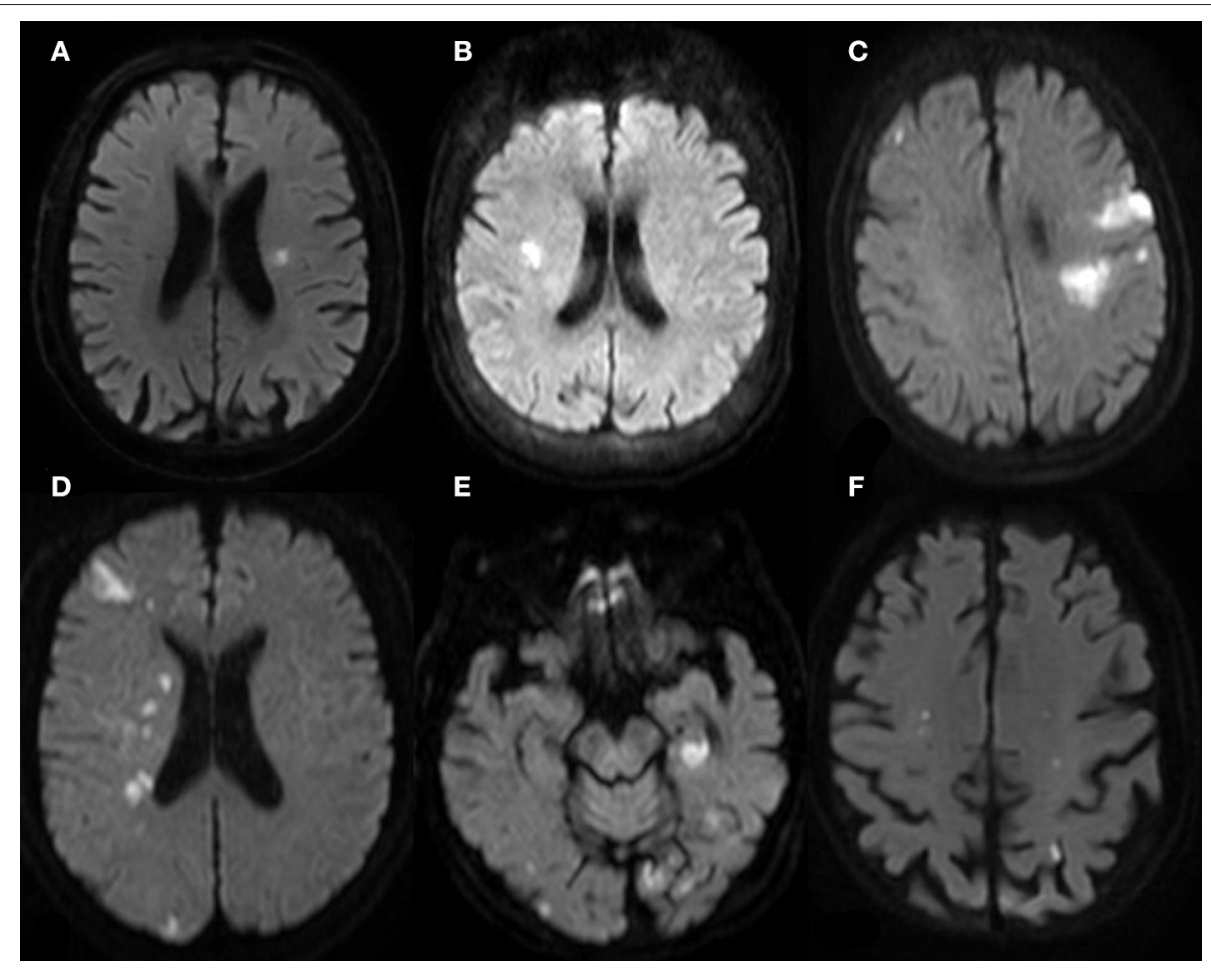

FIGURE 2 | MR (DWI) of patients with gastrointestinal malignant tumor-related AIS. (A,B) One Territory Sign (different patients): lesions with hyperintense signal in the supply area of LMCA in (A); lesions with hyperintense signal in the supply area of RMCA. (C,D) Two Territory Sign (different patients): multiple lesions in the supply area of LMCA and RACA in (C), involving cortex and deep white matter region; multiple lesions in the supply area of RMCA and RPCA in (D). (E,F) Three Territory Sign (the same patient): massive lesions in the left posterior hippocampus region supplied by the posterior choroid artery of LPCA; scattered lesions in bilateral occipital cortex and subcortex, more prominent on the left side. (E) shows two dotted lesions in the supply area of bilateral internal carotid arteries.

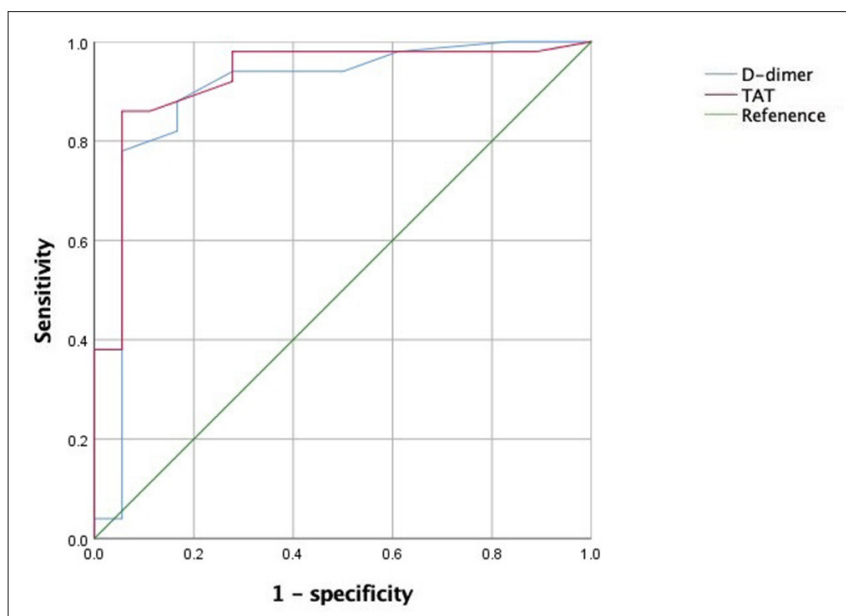

FIGURE 3 | ROC curve of TAT and D-dimer levels for prognosis in patients with gastrointestinal malignant tumor-related AIS.

\section{DISCUSSION}

Acute ischemic stroke may be identified prior to malignant tumor, which may be an early evidence of potential malignant tumor. Previous studies have found that patients with malignant tumor and with AIS as an initial symptom accounted for 0.4$3.0 \%$ of hospitalized stroke patients, and about $5.3-20 \%$ of latent stroke patients were diagnosed with malignant tumor during hospitalization, commonly including lung, breast, liver, stomach, and prostate cancers $(27,28)$. Malignant tumor-related AIS is prone to early neurological deterioration, with high recurrence and poor prognosis, and the prognosis varies in patients with different types of such cerebral infarctions (29). Based on the 2020 data from the International Agency for Research on Cancer (IARC), colorectal, liver, and gastric cancers were the third to fifth common malignant tumors in men, and colorectal cancer is the second common cancer in women (30). Analyzing the characteristics of gastrointestinal malignant tumor-related AIS is the basis for the treatment of such kinds of disease.

\section{Prognostic Factors in Patients With Gastrointestinal Malignant Tumor-Related AIS}

The results showed no significant difference between good and poor prognosis groups in common risk factors of AIS of TAT were 86.0 and $94.4 \%$, respectively. Figure 3 show ROC curve of TAT and D-dimer levels for prognosis in patients with gastrointestinal malignant tumor-related AIS. 
TABLE 1 | Univariate regression analysis of poor prognosis in patients with gastrointestinal malignant tumor-related AIS.

\begin{tabular}{|c|c|c|c|c|}
\hline Item & Total (68) & Good prognosis (18) & Poor prognosis $(50)$ & $P$-value \\
\hline Male, $n(\%)$ & 49 (72.06) & $14(77.78)$ & $35(70.00)$ & 0.528 \\
\hline Age, years & $61.78 \pm 6.65$ & $61.78 \pm 6.11$ & $61.78 \pm 6.90$ & 0.741 \\
\hline Hypertension, $n$ (\%) & $45(66.18)$ & $9(50.00)$ & $36(72.00)$ & 0.091 \\
\hline Diabetes, $n(\%)$ & $38(55.88)$ & 7 (38.89) & $31(62.00)$ & 0.090 \\
\hline Hyperlipidemia, $n$ (\%) & $43(63.24)$ & $9(50.00)$ & $34(68.00)$ & 0.174 \\
\hline Stroke, $n(\%)$ & $14(20.59)$ & $4(22.22)$ & $10(20.00)$ & 1.000 \\
\hline Smoking, $n(\%)$ & $24(35.29)$ & 6 (33.33) & $18(36.00)$ & 0.839 \\
\hline Drinking, $n(\%)$ & $34(50.00)$ & $10(55.56)$ & $24(48.00)$ & 0.582 \\
\hline Previous tumor history, $n$ (\%) & $52(76.47)$ & $11(61.11)$ & $41(82.00)$ & 0.574 \\
\hline MT to stroke after tumor, month & $6.15 \pm 3.21$ & $6.86 \pm 2.26$ & $5.64 \pm 3.02$ & 0.812 \\
\hline WBC (×109/L) & $7.01 \pm 2.10$ & $7.76 \pm 2.02$ & $6.74 \pm 2.08$ & 0.810 \\
\hline PLT (×109/L) & $314.22 \pm 20.85$ & $306.33 \pm 29.53$ & $317.06 \pm 16.14$ & 0.326 \\
\hline $\mathrm{Hb}, \mathrm{g} / \mathrm{L}$ & $119.09 \pm 10.34$ & $122.56 \pm 15.30$ & $117.84 \pm 7.69$ & 0.231 \\
\hline LDL-C, mmol/L & $4.11 \pm 0.72$ & $3.89 \pm 0.70$ & $4.19 \pm 0.72$ & 0.130 \\
\hline Hcy, $\mu \mathrm{mol} / \mathrm{L}$ & $18.35 \pm 3.21$ & $17.00 \pm 3.40$ & $18.84 \pm 3.03^{\mathrm{a}}$ & 0.047 \\
\hline Fib, g/L & $4.30 \pm 0.60$ & $4.18 \pm 0.57$ & $4.34 \pm 0.61$ & 0.981 \\
\hline D-dimer, mg/L & $1.89 \pm 0.85$ & $1.24 \pm 0.70$ & $2.12 \pm 0.78^{a}$ & $<0.001$ \\
\hline TAT, ng/ml & $6.93 \pm 3.98$ & $3.75 \pm 1.40$ & $8.07 \pm 3.99^{a}$ & $<0.001$ \\
\hline One territory sign & 7 (10.29) & $4(22.22)$ & $3(6.00)$ & 0.136 \\
\hline Two territory sign & 25 (36.76) & $4(22.22)$ & $21(42.00)$ & 0.136 \\
\hline Three territory sign & $30(44.12)$ & $4(22.22)$ & $26(52.00)^{a}$ & 0.029 \\
\hline
\end{tabular}

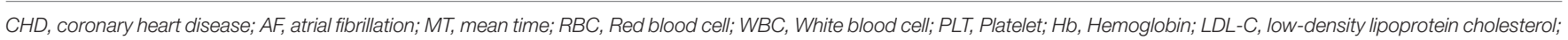
Hcy, homocysteine; Fib, fibrinogen; TAT, thrombin-antithrombin complex. ${ }^{a} P<0.05$, as compared to poor prognosis.

TABLE 2 | Multivariate analysis of poor prognosis in patients with gastrointestinal malignant tumor-related AIS.

\begin{tabular}{lccccccc}
\hline Variables & $\boldsymbol{\beta}$ & SE & Wals & Df & $\boldsymbol{P}$-value & OR & $\mathbf{9 5 \%} \mathbf{C l}$ \\
\hline Hcy & 0.259 & 0.164 & 2.493 & 1 & 0.114 & 1.296 & $0.939-1.788$ \\
D-dimer & 1.503 & 0.76 & 3.914 & 1 & 0.048 & 4.497 & $1.014-19.938$ \\
TAT & 1.457 & 0.487 & 8.957 & 1 & 0.003 & 4.294 & $1.654-11.149$ \\
Three territory & 2.2 & 1.216 & 3.271 & 1 & 0.071 & 9.021 & $0.832-97.827$ \\
sign & & & & & & & \\
\hline
\end{tabular}

including hypertension, coronary heart disease, diabetes, and hyperlipidemia. Higher levels of fibrinogen, TAT, and Ddimer, and greater proportion of patients with multiple scattered infarctions were observed in the poor prognosis group, which indicated a different mechanism of tumor-related AIS from the common atherosclerotic infarction. It has been proposed that tumor necrosis factors or cytokines produced by tumor cells can promote coagulation through DIC and form microthrombi (31). The mechanisms of malignant tumorrelated AIS include hypercoagulability, NBTE, infection, tumorrelated chemotherapy, endocrine therapy, and radiotherapy, among which, hypercoagulability plays the most important role. This study found that increased levels of D-dimer and TAT are independent prognostic factors of such patients, which is consistent with the results of previous studies (32). Results of this study also confirmed that hypercoagulation may be the major mechanism of cerebral infarction in patients with a malignant tumor.

\section{TAT as a Better Prognostic Factor Than D-Dimer in Tumor-Related AIS}

D-dimer has been proposed as a predictor of AIS in patients with a malignant tumor $(33,34)$. However, the limitations of the testing methods, interference by rheumatoid factor, immune complex, and intake of antitumor drugs or immunoenhancing drugs may lead to false positive results (35). With the updated testing methods, TAT and other indicators become more suitable for clinical application. These indicators are molecular markers of thrombosis and fibrinolysis that can reflect the state of coagulation and fibrinolysis in patients with a malignant tumor 
$(36,37)$. TAT is a complex formed by thrombin and antithrombin III. As a molecular marker of thrombin formation, TAT directly reflects the activation of the coagulation system. Increased TAT is a hint of procoagulant activation and inhibitor depletion and is one of the earliest indicators of coagulation dysfunction (38). Fidan et al. found that the level of TAT in gastric cancer patients was significantly higher than that in the control group, with stable expression at different tumor stages, which was a reliable marker for the hypercoagulation state of malignant tumors (39). The present study also confirmed that TAT expression was significantly correlated with the clinical prognosis in patients with gastrointestinal malignancy, and was more sensitive and specific than D-dimer. Therefore, we believe that TAT is better than D-dimer in predicting the prognosis of patients with malignant tumor-related thrombotic events, which is consistent with the results of Cui et al. (40).

\section{MRI Features of Gastrointestinal Malignant Tumor-Related AIS}

Previous research found that in AIS patients complicated with malignant tumor the incidence of infarction simultaneously involving three main intracranial arterial supply areas was six times that of patients with atrial fibrillation (41). A significant increase of D-dimer is a characteristic of malignant tumor-related AIS (42-44). In this study, most patients showed infarction in three territories on DWI (44.12\%), which was an independent risk factor for prognosis.

Echocardiogram was performed in all the patients to identify potential cardiogenic embolism, and $19.12 \%(13 / 68)$ of the patients were found to have NBTE which is basically consistent with the proportion of NBTE in about 19\% of patients with malignant tumors found by Edoute et al. (45). NBTE is most common in lung or gastrointestinal adenocarcinoma-related AIS associated with lung cancer or gastrointestinal adenocarcinoma, which is also reported in pancreatic and biliary cancer (46). However, NBTE was not found in patients with pancreatic and biliary cancer in this study.

\section{Prognosis, the Temporal Relation Between Gastrointestinal Malignant Tumor, and AIS}

It has been reported that the mortality of AIS was higher in patients complicated with malignant tumor than those without the tumor (47). The former research showed that a 3-month mortality rate of $46.9 \%$ in patients with malignant tumor complicated with AIS after receiving relevant treatment and $50 \%$ of the survivors had neurological sequela (48). Another study showed that an average follow-up of 29 months for 24 patients with cerebral infarction complicated with latent tumor, with mortality reaching 79\%. At a 1-year followup, $73.53 \%(50 / 68)$ of the patients showed poor outcomes (mRS > 2) including deaths (27). The poor therapeutic effects may be attributed to the complex mechanisms of cancer, or the onset of AIS leading to aggravation of the general condition of the patients, even affecting, or interrupting antitumor therapy.
Selvik et al. (49) found an average interval of 14 months from the onset of AIS, to the diagnosis of the tumor. In the present study, 16 patients (23.53\%) were diagnosed with malignant gastrointestinal tumor $12.39 \pm$ 4.12 months after AIS; 52 patients (76.47\%) developed AIS after the diagnosis of tumor, with an average interval of $6.15 \pm 3.21$ months, which is basically consistent with previous results showing an interval of 1.5-9 months (50).

\section{CONCLUSIONS}

Patients with AIS related to gastrointestinal malignant tumor have a relatively poor prognosis. Increased levels of TAT and Ddimer are independent risk factors for poor prognosis. TAT has a better performance than $\mathrm{D}$-dimer in predicting prognosis in patients with gastrointestinal malignant tumor-related AIS.

\section{LIMITATIONS}

Some limitations have to be admitted for we report a retrospective study with a relatively small number of patients. Due to the limited number of the included cases, tumor stage and treatment were not analyzed, which could limit the statistical significance of the results. Multicenter studies and larger sample are required for analysis to better summarize the clinical characteristics of malignant tumor patients complicated with AIS in future.

\section{DATA AVAILABILITY STATEMENT}

The raw data supporting the conclusions of this article will be made available by the authors, without undue reservation.

\section{ETHICS STATEMENT}

The studies involving human participants were reviewed and approved by Lanzhou University Second Hospital Ethics Committee. The patients/participants provided their written informed consent to participate in this study. Written informed consent was obtained from the individual(s) for the publication of any potentially identifiable images or data included in this article.

\section{AUTHOR CONTRIBUTIONS}

YLiu and XL contributed to the study design and drafted the manuscript. FS and XY analyzed the data in the literature and edited the manuscript. YLi contributed to the study design, supervised writing of the manuscript, critically reviewed the paper, and supervised the research project. All authors contributed to the article and approved the submitted version. 


\section{FUNDING}

This work was supported by grants from the Science and Technology Foundation for Young Scientist of Gansu Province, China (Grant no. 21JR1RA163), The Natural Science Foundation of Gansu Province, China (Grant no. 21JR1RA136), The Science and Technology Project of CuiYing, Lanzhou University Second Hospital, China (Grant no. CY2018-MS06), and

\section{REFERENCES}

1. Prandoni P, Falanga A, Piccioli A. Cancer and venous thromboembolism. Lancet Oncol. (2005) 6:401-10. doi: 10.1016/S1470-2045(05)70207-2

2. Noble S, Pasi J. Epidemiology and pathophysiology of cancer-associated thrombosis.Br J Cancer. (2010) 102 Suppl 1:S2-9. doi: 10.1038/sj.bjc.6605599

3. Levi M. Cancer-related coagulopathies. Thromb Res. (2014) 133 Suppl 2:S705. doi: 10.1016/S0049-3848(14)50012-6

4. Eichinger S. Cancer associated thrombosis: risk factors and outcomes. Thromb Res. (2016) 140 Suppl 1:S12-S17. doi: 10.1016/S0049-3848(16)30092-5

5. Grisold W, Oberndorfer S, Struhal W. Stroke and cancer: a review. Acta Neurol Scand. (2009) 119:1-16. doi: 10.1111/j.1600-0404.2008.01059.x

6. Kim SJ, Park JH, Lee MJ, Park YG, Ahn MJ, Bang OY. Clues to occult cancer in patients with ischemic stroke. PLOS ONE. (2012) 7:e44959. doi: 10.1371/journal.pone.0044959

7. Larsen AC, Dabrowski T, Frøkjær JB, Fisker RV, Iyer VV, Møller BK, et al. Prevalence of venous thromboembolism at diagnosis of upper gastrointestinal cancer. Br J Surg. (2014) 101:246-53. doi: 10.1002/bjs.9353

8. Kuan AS, Chen SC, Yeh CM, Hung MH, Hung YP, Chen TJ, et al. Risk of ischemic stroke in patients with gastric cancer: a nationwide population-based cohort study. Medicine. (2015) 94:e1336. doi: 10.1097/MD.0000000000001336

9. Collins RC, Al-Mondhiry H, Chernik NL, Posner JB. Neurologic manifestations of intravascular coagulation in patients with cancer. A clinicopathologic analysis of 12 cases. Neurology. (1975) 25:795-806. doi: 10.1212/WNL.25.9.795

10. Kim SG, Hong JM, Kim HY, Lee J, Chung PW, Park KY, et al. Ischemic stroke in cancer patients with and without conventional mechanisms: a multicenter study in Korea. Stroke. (2010) 41:798-801. doi: 10.1161/STROKEAHA.109.571356

11. Schwarzbach CJ, Schaefer A, Ebert A, Held V, Bolognese M, Kablau $\mathrm{M}$, et al. Stroke and cancer: the importance of cancer-associated hypercoagulation as a possible stroke etiology. Stroke. (2012) 43:3029-34. doi: 10.1161/STROKEAHA.112.658625

12. Dearborn JL, Urrutia VC, Zeiler SR. Stroke and Cancer-A Complicated Relationship. J Neurol Transl Neurosci. (2014) 2:1039.

13. Selvik HA, Thomassen L, Logallo N, Næss H. Prior cancer in patients with ischemic stroke: the Bergen NORSTROKE study. J Stroke Cerebrovasc Dis. (2014) 23:919-25. doi: 10.1016/j.jstrokecerebrovasdis.2013.07.041

14. Lee AY. Cancer and thromboembolic disease: pathogenic mechanisms. Cancer Treat Rev. (2002) 28:137-40. doi: 10.1016/S0305-7372(02)00044-0

15. Franco AT, Corken A, Ware J. Platelets at the interface of thrombosis, inflammation, and cancer. Blood. (2015) 126:5828. doi: 10.1182/blood-2014-08-531582

16. Peng Z, Shu B, Zhang Y, Wang M. Endothelial response to pathophysiological stress. Arterioscler Thromb Vasc Biol. (2019) 39:e233-43. doi: 10.1161/ATVBAHA.119.312580

17. Lefkovitz NW, Roessmann U, Kori SH. Major cerebral infarction from tumor embolus. Stroke. (1986) 17:555-7. doi: 10.1161/01.STR.17.3.555

18. Friedl P, Alexander S. Cancer invasion and the microenvironment: plasticity and reciprocity. Cell. (2011) 147:992-1009. doi: 10.1016/j.cell.2011.11.016

19. Elkind MS. Inflammatory mechanisms of stroke. Stroke. (2010) 41(10 Suppl):S3-S8. doi: 10.1161/STROKEAHA.110.594945

20. Ojeda VJ, Frost F, Mastaglia FL. Non-bacterial thrombotic endocarditis associated with malignant disease: a clinicopathological study of 16 cases. Med J Aust. (1985) 142:629-31. doi: 10.5694/j.1326-5377.1985.tb113555.x
Major Science and Technology Projects of Gansu Province, China (20ZD7FA003).

\section{SUPPLEMENTARY MATERIAL}

The Supplementary Material for this article can be found online at: https://www.frontiersin.org/articles/10.3389/fneur. 2021.777483/full\#supplementary-material

21. Zembower TR. Epidemiology of infections in cancer patients. Cancer Treat Res. (2014) 161:43-89. doi: 10.1007/978-3-319-04220-6_2

22. Bos MM, Smeets LS, Dumay I, de Jonge E. Bloodstream infections in patients with or without cancer in a large community hospital. Infection. (2013) 41:949-58. doi: 10.1007/s15010-013-0468-1

23. Saynak M, Cosar-Alas R, Yurut-Caloglu V, Caloglu M, Kocak Z, Uzal C. Chemotherapy and cerebrovascular disease. J BUON. (2008) 13:31-6.

24. Robinson D, Garmo H, Lindahl B, Van Hemelrijck M, Adolfsson J, Bratt O. et al. Ischemic heart disease and stroke before and during endocrine treatment for prostate cancer in PCBaSe Sweden. Int J Cancer. (2012) 130:478-87. doi: 10.1002/ijc.26022

25. Mosca L, Grady D, Barrett-Connor E, Collins P, Wenger N, Abramson BL, et al. Effect of raloxifene on stroke and venous thromboembolism according to subgroups in postmenopausal women at increased risk of coronary heart disease. Stroke. (2009) 40:147-55. doi: 10.1161/STROKEAHA.108. 518621

26. Scott AS, Parr LA, Johnstone PA. Risk of cerebrovascular events after neck and supraclavicular radiotherapy: a systematic review. Radiother Oncol. (2009) 90:163-5. doi: 10.1016/j.radonc.2008.12.019

27. Taccone FS, Jeangette SM, Blecic SA. First-ever stroke as initial presentation of systemic cancer. J Stroke Cerebrovasc Dis. (2008) 17:169-74. doi: 10.1016/j.jstrokecerebrovasdis.2008. 01.007

28. Ikushima S, Ono R, Fukuda K, Sakayori M, Awano N, Kondo K. Trousseau's syndrome: cancer-associated thrombosis. Jpn J Clin Oncol. (2016) 46:2048. doi: 10.1093/jjco/hyv165

29. Kneihsl M, Enzinger C, Wünsch G, Khalil M, Culea V, Urbanic-Purkart $\mathrm{T}$, et al. Poor short-term outcome in patients with ischaemic stroke and active cancer. J Neurol. (2016) 263:150-6. doi: 10.1007/s00415-0157954-6

30. Sung H, Ferlay J, Siegel RL, Laversanne M, Soerjomataram I, Jemal A, et al. Global Cancer Statistics 2020: GLOBOCAN Estimates of Incidence and Mortality Worldwide for 36 Cancers in 185 Countries. CA Cancer J Clin. (2021) 71:209-49. doi: 10.3322/caac.21660

31. Yigit M, Sogut O, Yigit E, Turkdogan KA, Kaplan O, Dur A, et al. The relationship between anemia and recurrence of ischemic stroke in patients with Trousseau's syndrome: a retrospective cross-sectional study. Turk J Emerg Med. (2016) 16:65-8. doi: 10.1016/j.tjem.2015. 11.013

32. Tsushima M, Metoki N, Hagii J, Saito S, Shiroto H, Yasujima M, et al. D-dimer and C-reactive Protein as Potential Biomarkers for Diagnosis of Trousseau's Syndrome in Patients with Cerebral Embolism. J Stroke Cerebrovasc Dis. (2020) 29:104534. doi: 10.1016/j.jstrokecerebrovasdis.2019. 104534

33. Chen W, He Y, Su Y. Multifocal cerebral infarction as the first manifestation of occult malignancy: case series of trousseau's syndrome and literature review. Brain Circ. (2018) 4:65-72. doi: 10.4103/bc.bc_1_18

34. Sorgun MH, Kuzu M, Ozer IS, Yilmaz V, Ulukan C, Cotur Levent H, et al. Risk Factors, Biomarkers, Etiology, Outcome and Prognosis of Ischemic Stroke in Cancer Patients. Asian Pac J Cancer Prev. (2018) 19:649-53. doi: 10.22034/APJCP.2018.19.3.649

35. Reitter EM, Kaider A, Ay C, Quehenberger P, Marosi C, Zielinski $\mathrm{C}$, et al. Longitudinal analysis of hemostasis biomarkers in cancer patients during antitumor treatment. J Thromb Haemost. (2016) 14:294305. doi: $10.1111 /$ jth. 13218 
36. Nakano $K$, Sugiyama $K$, Satoh $H$, Arifuku $H$, Fujimatsu $T$, Yoshida N, et al. Effect of Thrombomodulin Alfa on Disseminated Intravascular Coagulation in Patients with Lung Cancer. Intern Med. (2017) 56:1799-806. doi: 10.2169/internalmedicine. 56.7143

37. Lee SY, Niikura T, Iwakura T, Sakai Y, Kuroda R. Kurosaka M. Thrombin-antithrombin III complex tests. J Orthop Surg. (2017) 25:170840616684501. doi: 10.1177/0170840616684501

38. Kvolik S, Jukic M, Matijevic M, Marjanovic K, Glavas-Obrovac L. An overview of coagulation disorders in cancer patients. Surg Oncol. (2010) 19:e3346. doi: 10.1016/j.suronc.2009.03.008

39. Fidan E, Kavgaci H, Orem A, Yilmaz M, Yildiz B, Fidan S, et al. Thrombin activatable fibrinolysis inhibitor and thrombin-antithrombin-IIIcomplex levels in patients with gastric cancer. Tumour Biol. (2012) 33:151925. doi: 10.1007/s13277-012-0403-6

40. Cui CJ, Cui W, Gao J, Li J, Yan CE, Yu MY. TAT and PIC better than Ddimer in monitoring hypercoagulability of cancer patients. Chin J Lab Med. (2019) 42:853-7. doi: 10.3760/cma.j.issn.1009-8158.2019.10.008

41. Nouh AM, Staff I, Finelli PF. Three territory sign: an MRI marker of malignancy-related ischemic stroke (Trousseau syndrome). Neurol Clin Pract. (2019) 9:124-8. doi: 10.1212/CPJ.0000000000000603

42. Chi X, Zhao R, Pei H, Xing A, Hu S, Chen J, et al. Diffusion-weighted imagingdocumented bilateral small embolic stroke involving multiple vascular territories may indicate occult cancer: a retrospective case series and a brief review of the literature. Aging Med. (2020) 3:53-9. doi: 10.1002/agm2.12105

43. Selvik HA, Bjerkreim AT, Thomassen L, Waje-Andreassen U, Naess H, Kvistad CE. When to Screen Ischaemic Stroke Patients for Cancer. Cerebrovasc Dis. (2018) 45:42-7. doi: 10.1159/000484668

44. Prugger C, Luc G, Haas B, Morange PE, Ferrieres J, Amouyel $\mathrm{P}$, et al. Multiple biomarkers for the prediction of ischemic stroke: the PRIME study. Arterioscler Thromb Vasc Biol. (2013) 33:659-66. doi: 10.1161/ATVBAHA.112.300109

45. Edoute Y, Haim N, Rinkevich D, Brenner B, Reisner SA. Cardiac valvular vegetations in cancer patients: a prospective echocardiographic study of 200 patients. Am J Med. (1997) 102:252-8. doi: 10.1016/S0002-9343(96) 00457-3
46. Liu J, Frishman WH. Nonbacterial thrombotic endocarditis: pathogenesis, diagnosis, and management. Cardiol Rev. (2016) 24:244-7. doi: 10.1097/CRD.0000000000000106

47. Zhang YY, Cordato D, Shen Q, Sheng AZ, Hung WT, Chan DK. Risk factor, pattern, etiology and outcome in ischemic stroke patients with cancer: a nested case-control study. Cerebrovasc Dis. (2007) 23:1817. doi: $10.1159 / 000097639$

48. Cutting S, Wettengel M, Conners JJ, Ouyang B, Busl K. ThreeMonth Outcomes Are Poor in Stroke Patients with Cancer Despite Acute Stroke Treatment. J Stroke Cerebrovasc Dis. (2017) 26:809-15. doi: 10.1016/j.jstrokecerebrovasdis.2016.10.021

49. Selvik HA, Thomassen L, Bjerkreim AT, Næss H. Cancer-associated stroke: the Bergen NORSTROKE study. Cerebrovasc Dis Extra. (2015) 5:10713. doi: $10.1159 / 000440730$

50. Sørensen HT, Mellemkjaer L, Olsen JH, Baron JA. Prognosis of cancers associated with venous thromboembolism. N Engl J Med. (2000) 343:184650. doi: 10.1056/NEJM200012213432504

Conflict of Interest: The authors declare that the research was conducted in the absence of any commercial or financial relationships that could be construed as a potential conflict of interest.

Publisher's Note: All claims expressed in this article are solely those of the authors and do not necessarily represent those of their affiliated organizations, or those of the publisher, the editors and the reviewers. Any product that may be evaluated in this article, or claim that may be made by its manufacturer, is not guaranteed or endorsed by the publisher.

Copyright (c) $2021 \mathrm{Liu}, \mathrm{Li}$, Song, Yan, Han, Tang and Li. This is an open-access article distributed under the terms of the Creative Commons Attribution License (CC $B Y)$. The use, distribution or reproduction in other forums is permitted, provided the original author(s) and the copyright owner(s) are credited and that the original publication in this journal is cited, in accordance with accepted academic practice. No use, distribution or reproduction is permitted which does not comply with these terms. 